\title{
Competência Educativa Parental: Estudo de Propriedades Psicométricas da Escala com Amostra Brasileira
}

\author{
Maria Benedita Lima Pardo ${ }^{1}$ \\ Universidade Federal de Sergipe, São Cristóvão-SE, Brasil \\ Daniela Fonseca Freitas \\ Universidade do Porto, Porto, Portugal \\ Margarida Silveira Britto de Carvalho \\ Universidade Federal de Sergipe, São Cristóvão-SE, Brasil \\ Anne Marie Fontaine \\ Universidade do Porto, Porto, Portugal
}

\section{RESUMO}

O Questionário de Autoavaliação da Competência Educativa Parental (QACEP) é um instrumento que tem por objetivo avaliar a percepção de competência dos pais em suas funções. No Brasil, sua utilização é escassa e o objetivo desta pesquisa é analisar as propriedades psicométricas do QACEP. Foi utilizada uma amostra de 151 mães e pais, a maioria com nível de escolaridade até o ensino médio e renda até dois salários mínimos. A análise fatorial confirmatória do modelo proposto com dois fatores - a percepção de eficácia e de satisfação - revelou bom ajustamento global aos dados recolhidos. Análises posteriores revelam que a satisfação parental aumenta com a idade dos pais, com o nível de escolaridade e salário auferido. Tendo em conta os diversos processos de validação conduzidos, recomenda-se o uso dessa versão do QACEP na investigação e prática clínica, com pessoas de baixa renda no Brasil.

Palavras-chave: competência parental; eficácia; satisfação; análise fatorial confirmatória.

\section{ABSTRACT - Parental Educational Competence: Study of Psychometric Properties of the Scale with Brazilian Sample}

The Parenting Sense of Competence Scale (PSOC) is an instrument that aims to evaluate the perception of parental competence in its functions. In Brazil, it is scarcely used and the objective of this research is to analyze the psychometric properties of PSOC. A sample of 151 mothers and fathers was used, the majority with education level up to high school and income up to two minimum wages. Confirmatory factor analysis of a proposed two-factor model (perception of efficacy and satisfaction) revealed a good overall fit to the data collected. Later analyses revealed that parental satisfaction increases with the parents' age, with the level of schooling and income. Taking into account the different validation processes conducted, it is recommended to use this version of PSOC in research and clinical practice with low-income people in Brazil.

Keywords: parental competence; efficacy; satisfaction; confirmatory factor analysis.

RESUMEN - Competencia Educativa Parental: Estudio de Propiedades Psicométricas de la Escala con Muestra Brasileña

El Cuestionario de Autoevaluación de Competencia Educativa Parental (QACEP) es un instrumento que tiene por objetivo evaluar la percepción de competencia de los padres en sus tareas. En Brasil, su utilización es escasa y el objetivo de esta investigación es analizar las propiedades psicométricas del QACEP. Se utilizó una muestra de 151 madres y padres, la mayoría con nivel de escolaridad hasta Enseñanza Secundaria y una renta media familiar hasta dos salarios mínimos. El análisis factorial confirmatorio del modelo propuesto con dos factores - Eficacia y Satisfacción - mostró buen ajuste global a los datos recogidos. Análisis posteriores revelan que la satisfacción parental aumenta con la edad de los padres, con el nivel de escolaridad y con los ingresos familiares. Se recomienda el uso de esta versión del QACEP en la investigación y práctica clínica con personas de bajos ingresos en Brasil.

Palabras clave: competencia parental; eficacia; satisfacción; análisis factorial confirmatorio.

De forma global, a competência tem sido definida como a capacidade de as pessoas agirem efetivamente no ambiente (Wagner \& Morse, 1975). As ações efetivas apresentam um lado subjetivo identificado como sentido de competência ou a percepção que a pessoa tem sobre suas habilidades e que reflete a confiança resultante das interações com o ambiente ao longo da vida. A ação competente resulta em importantes recompensas psicológicas

${ }^{1}$ Endereço para correspondência: Universidade Federal de Sergipe. Departamento de Psicologia. Rodovia Marechal Rondon, s/n, 49000-000, São Cristóvão-SE. E-mail: pardombl@hotmail.com

As autoras agradecem a colaboração das alunas do curso de Psicologia, Katiane dos Santos Costa, Sabrina Libório Fontes e Thayná dos Santos Pinheiro, na coleta de dados e lançamento no banco de dados. Apoio institucional PIBIX/PROEX/UFS. 
que incentivam o indivíduo a continuar se comportando. Os autores chamam a atenção para essas recompensas, pois a autoestima e a estima atribuída pelos outros à pessoa são contingentes às habilidades de dominar o meio e, em consequência, ao próprio sentido de competência. Assim o interesse pelo estudo desse fenômeno se assenta em sua possível influência sobre as ações dos indivíduos.

A percepção de competência pode estar presente em diferentes áreas de atuação do indivíduo e uma pessoa pode perceber-se competente em uma área e não em outra (Trudelle \& Montambault, 1994). A competência parental diz respeito, especificamente, à percepção do desempenho do papel de pai ou de mãe e encontra-se em parte dependente das condições de contexto que asseguram sua atuação como cuidador. A existência de fatores além dos contextuais é evidenciada com base na observação que, mesmo em circunstâncias semelhantes, observa-se uma ampla variação nas manifestações dessa competência.

O estudo da competência parental reveste-se de grande interesse prático. Pesquisas têm apontado que as crenças de eficácia estão associadas a práticas parentais positivas (Coleman \& Karraker, 1997, 2000). Estudo de revisão (Jones \& Prinz, 2005) apontou a necessidade de estudos experimentais e longitudinais que clarifiquem a direção causal dessas dimensões. Pesquisadores também têm estudado os diferentes fatores que podem contribuir para a felicidade ou bem-estar dos pais (Nelson, Kushlev, \& Lyubormirsky, 2014). Trudelle e Mountambault (1994) referem-se à hipótese de que as pessoas que se sentem bem na função parental, e se percebem como bons pais, desempenham seus papéis de modo mais eficaz. Contudo, são necessários estudos longitudinais que demonstrem a existência de uma causalidade específica na relação entre essas variáveis.

Para avaliar a eficácia da intervenção em termos de competência parental faltam, no Brasil, instrumentos cuja consistência e validade sejam evidenciados. Em outras culturas, a literatura mostra o interesse prático neste tipo de instrumento através de pesquisas que estudam os componentes da competência parental e que encontraram correlação positiva com a auto-estima dos pais (Afonso, 2011), o estilo parental positivo (Ohan, Leung e Johnston, 2000), a idade e a educação parental (Gilmore \& Cuskelly, 2008). Por outro lado, Grady e Karraker (2016) verificaram que o temperamento negativo da mãe e da criança podem ser preditores da avaliação negativa do senso de competência parental. Em todos esses estudos, os autores indicam a necessidade de continuidade de pesquisas que clarifiquem as relações entre a percepção da competência e o modo de agir dos pais.

\section{O Questionário de Autoavaliação de Competência Educativa Parental (QACEP)}

Um dos instrumentos que tem sido muito utilizado para medir a autopercepção de pais é o Questionário de Autoavaliação da Competência Educativa Parental (QACEP). Esse instrumento foi apresentado por GibaudWallston e Wandersman, em 1978 (como citado em Johnston \& Mash, 1989) com a denominação de Escala do Sentido de Competência Parental (Parenting Sense of Competence Scale, PSOC). A escala era constituída por 17 itens que se distribuíam por dois fatores Habilidade/ Conhecimento e Valor/Conforto. O primeiro fator dizia respeito ao conhecimento ou domínio do pai ou da mãe sobre ações relacionadas às funções parentais. $\mathrm{O}$ segundo fator referia-se à avaliação dos pais sobre sua satisfação no exercício dessas funções. Inicialmente, essa escala foi utilizada para medir a percepção de competência de mães cujos bebês haviam nascido recentemente (Cutrona \& Troutman, 1986). Em 1989, Johnston e Mash ampliaram sua utilização para pais de crianças entre quatro e nove anos e realizaram um estudo sobre as propriedades psicométricas. A escala foi reduzida para 16 itens. Foi descartado o item Q17 "Ser um bom pai/mãe é recompensador" por não ter saturado em nenhum dos fatores e o item Q8 "É difícil para um pai (mãe) saber se aquilo que ele faz com o seu filho é bom ou mau" foi transposta para o fator Satisfação. Essas autoras também renomearam os fatores que a compunham. O fator Habilidade/Conhecimento passou a ser denominado de Eficácia, contendo sete itens, e o fator Valor/Conforto passou a ser denominado de Satisfação, contendo nove itens. A consistência interna das subescalas obteve os valores de 0,76 para a Eficácia e 0,75 para a Satisfação.

O QACEP tem sido aplicado em diversos países (e.g., Estados Unidos da América, Canadá, Austrália, Portugal, China) e não foram todos os pesquisadores que adotaram a nova versão da escala. Há um grupo de investigadores que tem utilizado a versão original de 17 itens (PSOC) de Gibaud-Wallston e Wandersman (1978, como citado em Johnston \& Mash, 1989) e outro grupo que utiliza a versão proposta por Johnston e Mash (1989), com 16 itens. Terisse e Trudelle (1988) traduziram para o francês a escala de 16 itens, realizaram sua adaptação para o Canadá e a intitularam "Questionnaire d'auto-evaluation de la competence éducative parentale" (QACEP).

A estrutura fatorial estudada do QACEP foi explorada por diferentes autores. Essas pesquisas têm encontrado duas soluções diferentes. Há autores que consideram adequada a estrutura original com dois fatores, com as subescalas de Eficácia e Satisfação (Afonso, 2011; Gomes-da-Costa et al., 2006; Johnston \& Mash, 1989; Ngai, Chan, \& Holroyd, 2007; Ohan et al, 2000; SeabraSantos et al., 2015). Os dois fatores que compõem o QACEP, representam os aspectos instrumental (Eficácia) e emocional (Satisfação) do exercício das funções parentais. Já outros autores, em menor número, consideram mais adequada uma estrutura de três fatores, incluindo também uma subescala denominada Interesse, com apenas dois itens (Ferreira, Veríssimo, Santos, Fernandes, \& Cardoso, 2011; Gilmore \& Cuskelly, 2008; Rogers \& 
Matthews, 2004). Com dois fatores, os índices de consistência interna, avaliada pelo alpha de Cronbach, são satisfatórios, a saber: entre .61 e .80 para a subescala de Eficácia, entre .72 e .83 para a de Satisfação. Na solução com três fatores, o terceiro, denominado de Interesse, demonstrou valores de confiabilidade baixos na maioria dos estudos, a saber: .57 (pais) e .62 (mães) no estudo de Gilmore e Cuskelly (2008), .62 (pais) e .58 (mães) no de Rogers e Mathews (2004), com exceção para o de Ferreira et al. (2011) que obteve índice de .82 para este fator.

Como apontam Meunier e Roskam (2009), a maioria dos estudos sobre a competência parental tem sido desenvolvidos com pessoas que falam inglês e que pertencem à classe média. As escalas precisam ser validadas em cada cultura e, no Brasil, encontra-se apenas um estudo que utilizou o QACEP (Silva \& Aiello, 2009). Essas autoras utilizaram o instrumento com 17 itens com pais de crianças com deficiência mental. Contudo, nesse estudo, não foi avaliada a estrutura fatorial e as propriedades psicométricas dessa escala. Em nosso país também não há estudos deste instrumento com populações de baixa renda. A necessidade de adaptação de instrumentos para tais populações foi apontada por autores que trabalharam com famílias em situação de risco (Menéndez, Giménez \& Hidalgo, 2011; Nunes, Jiménez, Menéndez, AyalaNunes, \& Hidalgo, 2016).

\section{Competência parental e suas associações com as dimensões sociodemográficas}

A literatura tem mostrado que, comparando as duas dimensões da escala, os níveis de satisfação costumam ser mais altos do que os de eficácia (Ohan et al., 2000; Gomes-da-Costa et al., 2006). Estudos também têm mostrado que o gênero influencia nestas percepções. Escores mais altos de satisfação têm sido obtidos para os pais (Johnston \& Mash, 1989) e de eficácia para as mães (Gilmores \& Cuskely, 2009). Já a pesquisa de Gomes-daCosta et al. (2006) mostrou que tanto pais quanto mães avaliaram-se como mais satisfeitos do que eficazes em suas funções.

A percepção de competência parental encontra-se ainda associada a outras dimensões de ordem sociodemográfica. Observou-se que a satisfação no desempenho das tarefas parentais correlaciona-se positivamente com o nível educacional dos pais (Gilmores \& Cuskely, 2009; Seabra-Santos et al., 2015). No estudo de Trudelle e Montambault (1994), os resultados indicaram que à medida que a idade dos filhos aumenta, o sentimento de competência das mães e dos pais decresce. Observou-se também que tanto para mães como para pais o nível da ocupação estava negativamente correlacionado com a avaliação global da competência, ou seja, quanto mais elevado era o nível da ocupação menos competentes se sentiam.

Tendo sido, a maioria das pesquisas, conduzidas com amostras de diferentes países é necessário o estudo das propriedades da escala quando aplicada a grupos brasileiros. Neste contexto haverá diferenciação nos níveis de eficácia e satisfação avaliados pelos pais? A avaliação dessas dimensões sofrerá influência da idade, da escolaridade dos pais e do rendimento familiar?

Esta pesquisa tem o objetivo de analisar as propriedades psicométricas do QACEP em relação a uma amostra de mães e pais brasileiros de baixa renda, testando a adequação de sua estrutura em relação ao modelo teórico proposto. Adicionalmente serão analisadas as variações dos níveis de satisfação e eficácia apurados pela QACEP, em função de aspectos sociodemográficos, como a idade dos pais, a escolaridade e a renda familiar.

\section{Método}

\section{Participantes}

A amostra é composta por 151 participantes, sendo $138(91,4 \%)$ mães ou pais biológicos, seis (4,0\%) mães ou pais adotivos e seis $(4,0 \%)$ avós. O número de mulheres respondentes foi de $128(84,8 \%)$ e o de homens 23 $(15,2 \%)$. A média das idades foi de 37,1 anos $(D P=8,07)$ e os participantes foram categorizados em dois grupos, com tamanho similar, um com idades entre 21 e 35 anos $(n=69 ; 47,3 \%)$ e o outro com idades entre 36 e 64 anos $(n=77 ; 52,7 \%)$. Em termos da escolaridade observa-se que $28,2 \%(n=42)$ concluiu o ensino fundamental, $45,6 \%(n=68)$ concluiu o ensino médio e $26,2 \%(n=39)$ tem habilitações de nível superior. Quanto ao ganho salarial, 38,4\% $(n=56)$ auferiam até o valor de um salário mínimo, 45,2\% $(n=66)$ recebiam valores entre um e dois salários mínimos e apenas $16,4 \%(n=24)$ tinham um rendimento superior a dois salários mínimos, o que permite situá-los nos estratos de renda familiar inferiores (C2, D, E) de acordo com o Critério Brasil (Associação Brasileira de Empresas de Pesquisa, 2016). No que concerne à ocupação profissional, segundo a Classificação Brasileira de Ocupações (Brasil, 2010), 95 pessoas (62,9\%) tinham ocupação de nível elementar, 29 (19,2\%) ocupação de nível médio e 18 (11,9\%) ocupação de nível superior.

\section{Instrumento}

Questionário de Autoavaliação da Competência Educativa Parental (QACEP) utilizado nesta pesquisa foi obtido a partir da tradução portuguesa realizada por Gomes-da-Costa et al. (2006), que se basearam na adaptação de Terisse e Trudelle (1988). Esses autores utilizaram a escala com 16 itens. Vários outros estudos também excluíram o item Q17 (Gomes-da-Costa et al., 2006; Ferreira et al., 2011; Ohan et al., 2000; SeabraSantos et al., 2015) pelo fato de não saturar em nenhum dos fatores previstos e pela dificuldade em enquadrá-la teoricamente nesses fatores.

No âmbito do processo de adaptação, foi feita a adequação da linguagem para o português do Brasil por duas pesquisadoras com experiência no atendimento a pais em 
serviço público. Em seguida, o questionário foi enviado a três especialistas que trabalhavam com Psicologia do Desenvolvimento ou adaptação de instrumentos, a fim de que analisassem a equivalência idiomática e experiencial da linguagem utilizada, tendo em vista a população a que se destinava. Nesse procedimento, seguiram-se as orientações de Borsa, Damásio e Bandeira (2012). Os especialistas sugeriram algumas mudanças, nomeadamente: trocar a expressão "tomando-me como exemplo" para "seguindo meu exemplo" no item Q6; alterar o enunciado do item Q7 de modo a fortalecer a conexão entre as duas afirmações colocadas, "Aprendendo a ser pai (mãe), a maior parte dos problemas da educação são fáceis de resolver"; e substituir termos no item Q10 como "habilidades" e "anseios" por outros de uso mais comum como "o que tenho aprendido" e "aquilo que desejei para mim". Em seguida, a fim de verificar a validade aparente, o instrumento foi alvo de uma reflexão falada junto de dez mães que apresentavam características semelhantes às da população com a qual se pretendia trabalhar, mais precisamente, apresentavam renda entre um e três salários mínimos e possuíam pelo menos um filho com idade entre um ano e meio e doze anos. Nesse processo, não foram sugeridas novas alterações.

Cada item da QACEP foi respondido de acordo com uma escala de tipo Likert que varia de 1 (estou totalmente de acordo) a 6 (estou totalmente em desacordo). Um exemplo de item da subescala de Eficácia é "Acredito que tenho todas as habilidades necessárias para ser um bom pai (mãe)", e da subescala de Satisfação é "Às vezes me sinto incapaz de controlar a situação com meu filho". Para se obter a avaliação do sentimento de competência, deve-se somar os valores das duas subescalas, invertendo-se os valores dos itens Q1, Q6, Q7, Q10, Q11, Q13 e Q15 (subescala de Eficácia), a fim de, para todos os itens, escores maiores indiquem maior sentimento de competência.

\section{Procedimentos de coleta de dados}

A presente pesquisa foi aprovada pelo Comitê de Ética da Universidade na qual foi realizada (CAAE 62322016.6.0000.5546) e respeitou todos os princípios recomendados pela Resolução 466/2012 do Conselho Nacional de Saúde para pesquisas com seres humanos. A aplicação foi feita em quatro escolas públicas da cidade de Aracaju e nas dependências da Universidade Federal de Sergipe. Nas escolas, os participantes foram contatados no decorrer de reuniões de pais convocadas rotineiramente. As pesquisadoras explicavam o objetivo da pesquisa, identificar modos de pensar de pais sobre a educação de filhos e solicitavam a anuência em participar. O questionário era anônimo e a participação voluntária. Quando não havia reunião agendada, os pais eram contatados no ambiente da escola pessoalmente pelas pesquisadoras, sendo o procedimento de aplicação e de recolhimento do material o mesmo. Em uma das escolas, a diretora se dispos a entregar pessoalmente os questionários aos pais e recolher os que foram respondidos. Os critérios para participação na pesquisa eram possuir pelo menos um filho com idade entre um ano e meio e doze anos e assinar o Termo de Consentimento Livre e Esclarecido (TCLE).

\section{Análise de dados}

Com base no fato de, nas pesquisas que encontraram solução com três fatores, o terceiro fator - interesse - ser constituído por um número pequeno de itens e também por apresentar menor confiabilidade que os demais, optou-se por realizar a análise fatorial confirmatória (AFC) da escala, respeitando a estrutura original proposta, ou seja, avaliou-se a adequação da estrutura com dois fatores, com os fatores Eficácia e Satisfação (Johnston \& Mash,1989). Caso os índices de ajustamento não fossem considerados bons, proceder-se-ia à análise fatorial exploratória.

Para avaliar a validade fatorial da escala, começou-se por uma análise da curva da distribuição dos resultados e pela consideração dos valores absolutos de assimetria e curtose. Itens com elevado desvio à distribuição da curva normal $(s k<2, k u<5$; Kline, 2004 como citado em Marôco, 2010) foram excluídos. Com recurso ao software AMOS (v.22), realizou-se a análise fatorial confirmatória (AFC), utilizando o método da máxima verosimilhança. Para apreciação da qualidade global do ajustamento do modelo, consideraram-se os valores do qui-quadrado ajustado $(\chi 2 / g l<2)$, do Bentler Comparative Fit Index (CFI $>0.90)$, do Root Mean Square Error of Approximation (RMSEA <0,08; $p>0,05)$, em consonância com as indicações citadas em Marôco (2010). Para assegurar a validade fatorial do modelo, analisaram-se os coeficientes de regressão estandardizados $(\lambda>0,50)$ e os correspondentes coeficientes de fiabilidade dos itens $\left(\lambda^{2}>0,25\right)$. Adicionalmente, a validade convergente foi considerada por meio do cálculo da variância extraída média $(V E M>0,5)$. Para a fiabilidade do fator, considerou-se o indicador de fiabilidade compósita $(\mathrm{FC}>0,70)$ e a consistência interna avaliada pelo alfa de Cronbach $(\alpha>0,7)$. Adicionalmente, considerou-se que havia validade discriminante quando os valores de VEM eram superiores ao quadrado do valor da correlação de Pearson entre os fatores (Marôco, 2010).

A fim de medir o grau de correlação entre a escala original e a escala obtida após a realização da AFC foi feita a correlação de Pearson. Para analisar a associação entre a idade e as dimensões da competência parental realizou-se uma correlação e análises de variância (ANOVAs) comparando as médias dos $50 \%$ dos participantes com menor idade e os de maior idade. Foram ainda realizadas ANOVAs em função do nível de escolaridade e da renda. Nessas análises, foi avaliado o tamanho do efeito da variável por meio do eta quadrado $\left(\eta^{2}\right)$ e das diferenças entre as médias pelo $d$ de Cohen, sendo a interpretação realizada de acordo com a literatura e os valores recomendados para as ciências sociais (Ferguson, 2009). Nas variáveis 
com mais de três grupos, quando houve diferenças significativas, foram realizados testes post hoc Tukey ou Games-Howell (consoante a verificação do pressuposto da homogeneidade das variâncias) a fim de identificar entre quais grupos ocorria a diferença.

\section{Resultados}

A análise preliminar à distribuição dos itens revelou que o item Q12, relacionado à dimensão satisfação parental, apresentou um valor de curtose alto $(\mathrm{ku}=5,44)$. Dado que esse item não pareceu discriminar bem os participantes, decidiu-se pela não inclusão dele na AFC. Os itens restantes apresentaram valores absolutos de assimetria e curtose mais próximos de uma distribuição normal $(|s k|<1,58,|k u|<1,67)$.

Os índices de ajustamento da primeira AFC apresentaram-se bons, revelando uma boa correspondência entre o modelo estrutural testado e os dados recolhidos, $\chi^{2} / g l=1,255, \mathrm{CFI}=0,915 ; \mathrm{RMSEA}=0,041, p_{\text {RSMEA }}=0,719$. Contudo, a nível do ajustamento local, observou-se que alguns itens apresentavam coeficientes de regressão estandardizados abaixo do ideal de 0,50 , o que indica uma fraca relação do item com a variável latente, sendo baixa a variância explicada (menor de 25\%). Desse modo, procedeu-se com a exclusão dos itens com baixos coeficientes de regressão estandardizados de forma sequencial, até se manter apenas os itens com coeficientes superiores a 0,40 . Os itens excluídos foram os seguintes; Q13 $(\lambda=0,20)$, Q7 $(\lambda=0,23), \mathrm{Q} 1(\lambda=0,29), \mathrm{Q} 5$ $(\lambda=0,34), \mathrm{Q} 8(\lambda=0,38)$. Após a retirada desses, itens os índices de ajustamento global melhoraram ligeiramente, $\chi^{2} / g l=1,188, \mathrm{CFI}=0,970, \mathrm{RMSEA}=0,035, p_{\text {RSMEA }}=0,703$. Considerando que os resultados da análise confirmatória chegaram a um ajustamento muito bom, as subescalas ficaram assim constituídas: Eficácia composta por quatro itens, Q6, Q10, Q11, Q15 e Satisfação composta por seis itens, Q2, Q3, Q4, Q9, Q14, Q16. A Figura 1 mostra esse resultado da análise fatorial confirmatória.

Os fatores apresentam uma baixa variância extraída média (VEM), sendo a VEM da Eficácia de 0,36 e a VEM da Satisfação de 0,31. Esse resultado pouco satisfatório revela a baixa validade convergente da escala e deve-se sobretudo aos relativamente baixos coeficientes de regressão estandardizados observados em todos os itens. A fiabilidade compósita (FC) dos fatores é adequada, sendo FC Eficácia =0,68, FC Satisfação=0,73. Avaliando a confiabilidade com o alfa de Cronbach, os valores observados são de 0,66 para a subescala de Eficácia e 0,73 para a subescala de Satisfação. A validade discriminante das subescalas está assegurada, pois estas não se encontram significativamente correlacionadas, $r^{2}=0,045, p=0,746$.

$$
\begin{gathered}
\text { AFC }- \text { QACEP } \\
\chi^{2}(34)=40,396 ; p=, 209 ; \chi^{2} / d f=1.188 \\
\text { CFI }=0,970 ; \text { PCFI }=0,599 ; \text { RMSEA }=0,035 ; p_{\text {RMSEA }}=0,703
\end{gathered}
$$

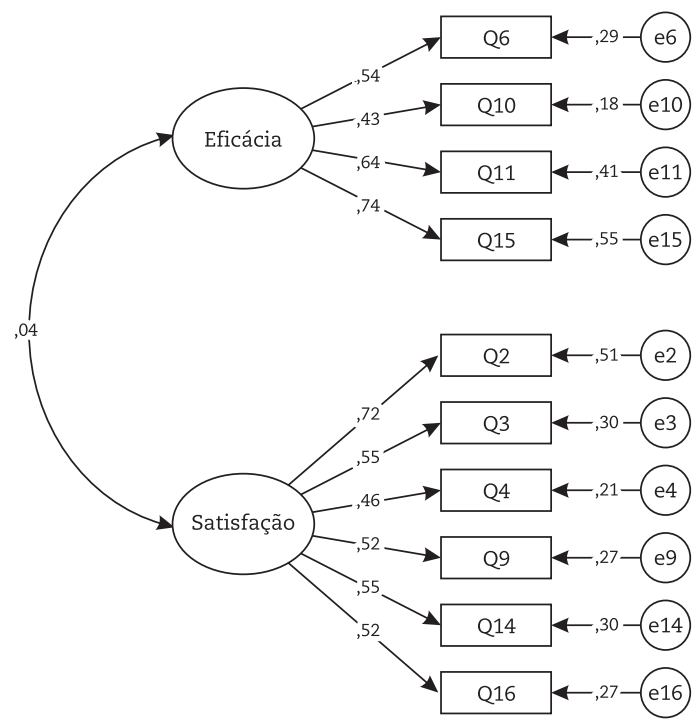

Figura 1. Análise fatorial confirmatória do QACEP

De forma a facilitar a leitura dos resultados com um menor número de itens, optou-se por calcular o escore total de cada fator recorrendo à média aritmética. Para confirmar se as características da escala reduzida se assemelhavam às da escala original, foi calculada a correlação entre a escala obtida após a AFC e a escala original com todos os itens. A correlação de Pearson mostrou-se muito alta e significativa para as subescalas da Eficácia, $r(151)=0,87, p<0,001$, assim como, para a de Satisfação $r(151)=0,95, p<0,001$. Esse resultado indica que a 
ordenação dos participantes em função dos seus níveis de Eficácia e Satisfação é extremamente similar nas escalas reduzidas e original.

O teste $t$ para amostras emparelhadas revelou uma diferença significativa entre as médias obtidas nas dimensões eficácia e satisfação, $t(150)=5,68, p<.001$. A média da eficácia mostrou-se significativamente mais elevada
$(M=4.84 ; D P=1.04)$ do que a da satisfação $(M=4.11$; $D P=1.17)(d$ de Cohen $=0.46)$.

De modo a verificar se a Eficácia e a Satisfação estão relacionadas com as características sociodemográficas, realizaram-se correlações e ANOVAs para as faixas de idade, escolaridade e nível salarial. Os resultados dessa análise estão na Tabela 1.

Tabela 1

Médias, Desvios Padrão e ANOVAs para Eficácia e Satisfação por Faixas de Idade, Escolaridade e Salário

\begin{tabular}{|c|c|c|c|c|}
\hline & \multicolumn{2}{|c|}{ Eficácia } & \multicolumn{2}{|c|}{ Satisfação } \\
\hline & M & DP & M & DP \\
\hline Idade & \multicolumn{2}{|c|}{$r=0,00, p=0,975$} & \multicolumn{2}{|c|}{$r=0,04, p=0,601$} \\
\hline $21-35$ & 4,78 & 1,03 & $3,89 \mathrm{a}$ & 1,14 \\
\hline \multirow[t]{2}{*}{$36-64$} & 4,88 & 1,04 & $4,36 \mathrm{~b}$ & 1,14 \\
\hline & \multicolumn{2}{|c|}{$F(1,144)=0,32, p=0,573, \eta^{2}=0,002$} & \multicolumn{2}{|c|}{$F(1,144)=6,09, p=0,015, \eta^{2}=0,041$} \\
\hline \multicolumn{5}{|l|}{ Escolaridade } \\
\hline N. Fundamental & 4,95 & 1,18 & $3,67 \mathrm{a}$ & 1,23 \\
\hline N. Médio & 4,89 & 0,92 & $4,10 \mathrm{a}$ & 1,13 \\
\hline \multirow[t]{2}{*}{ N. Superior } & 4,65 & 1,04 & $4,66 \mathrm{~b}$ & 0,95 \\
\hline & \multicolumn{2}{|c|}{$F(2,146)=1,06, p=0,350, \eta^{2}=0,014$} & \multicolumn{2}{|c|}{$F(2,146)=8,04, p=0,001, \eta^{2}=0,099$} \\
\hline \multicolumn{5}{|l|}{ Salário } \\
\hline Menos de 1 S.M. & 4,90 & 1,21 & $3,68 \mathrm{a}$ & 1,21 \\
\hline Entre 1 a 2 S.M & 4,94 & 0,80 & $4,26 \mathrm{~b}$ & 1,14 \\
\hline \multirow[t]{2}{*}{ Mais de 2 S.M. } & 4,56 & 0,98 & $4,78 \mathrm{c}$ & 0,71 \\
\hline & \multicolumn{2}{|c|}{$F(2,143)=1,33 p=0,269, \eta^{2}=0,018$} & \multicolumn{2}{|c|}{$F(2,143)=90,03, p=0,001, \eta^{2}=0,112$} \\
\hline
\end{tabular}

Nota. Letras diferentes indicam médias significativamente diferentes

As correlações não revelaram uma associação significativa entre a idade e as variáveis da competência parental. Contudo, os resultados das ANOVAs mostram que as médias na satisfação são significativamente diferentes, sendo que os pais mais velhos (de 36 a 55 anos) apresentaram maior satisfação do que os pais mais novos (21 a 35 anos) $(p<0,015)$. No entanto, a magnitude dessa diferença é de tamanho reduzido ( $d$ de Cohen $=0,41)$. Não se observaram diferenças estatisticamente significativas nos níveis de eficácia para os grupos de diferentes idades.

Em relação aos grupos de escolarização, não houve diferenças significativas para a eficácia. Novamente, observaram-se diferenças significativas em relação a satisfação. O teste de post hoc Tukey HSD mostrou que as diferenças significativas ocorreram entre o grupo de pais com escolarização de nível superior e o grupo com o nível fundamental $(p<0,001 ; \mathrm{d}$ de Cohen $=0,90)$ e com o nível de escolarização médio $(p<0,029 ; d$ de Cohen $=0,53)$. Não houve diferenças significativas entre os pais com ensino médio e os que possuem ensino fundamental $(p<0,122)$.

Considerando as faixas salariais, a ANOVA revelou que não há diferenças significativas na dimensão da eficácia. No entanto, na satisfação tais diferenças foram observadas. Os resultados indicaram diferenças significativas na satisfação entre as três faixas salariais.
Especificamente, observou-se que os pais que ganhavam acima de dois salários mínimos sentiam-se mais satisfeitos do que os pais que ganhavam entre um e dois salários mínimos $(p<0,032, d$ de Cohen $=0,50)$ e que os pais que ganhavam menos que o salário mínimo $(p<0,001, d$ de Cohen $=1,00)$. Por sua vez, os pais que ganhavam entre um e dois salários apresentaram maior satisfação do que os estavam na faixa salarial inferior ao salário mínimo $(p<0,024, d$ de Cohen=0.49).

\section{Discussão}

Esta pesquisa teve como um dos objetivos estudar as propriedades psicométricas do QACEP em uma amostra de pais brasileiros de baixa renda. O estudo foi desenvolvido a partir da necessidade de se adaptar um instrumento que auxiliasse na avaliação dos resultados de grupos de intervenção com pais, a serem desenvolvidos com esta população. Nesse contexto, as coletas de dados foram realizadas com uma amostra de pais brasileiros cujas características se assemelhassem às dessa população. A maioria dos participantes possuía renda familiar correspondente aos estratos socioeconômicos inferiores e escolaridade predominantemente nos níveis elementar e médio. Escolheu-se o QACEP por ser um instrumento 
amplamente utilizado e que apresentou boas qualidades psicométricas quando aplicado a grupos de outros países.

\section{A Adaptação da Escala}

A análise fatorial confirmatória revelou que a estrutura da escala tem um ajustamento muito bom aos dados da amostra. Contudo, houve necessidade de descartar um certo número de questões da escala original, devido a sua fraca relação com as correspondentes dimensões latentes. A exclusão desses itens mostrou-se necessária para assegurar a validade fatorial do instrumento, tornando-o mais robusto e a sua aplicabilidade mais confiável. Previamente, outros estudos já haviam apresentado que alguns itens têm baixos pesos fatoriais na escala, denunciando a pertinência de estes serem excluídos nas avaliações. A título de exemplo, Gilmores e Cuskely (2008) precisaram descartar o item Q5 (que não saturou em nenhum fator na análise fatorial exploratória) e os itens Q1 e Q7 (para obter uma boa consistência interna da escala global). Também Ohan et al. (2000) verificaram que, na análise fatorial exploratória, o item Q5 apresentou um peso fatorial abaixo de 0,40 para os pais, o mesmo acontecendo com o item Q12 para as mães. Por outro lado, no estudo de Ngai et al. (2007), onde não houve exclusão de itens, os coeficientes de regressão estandardizados da análise factorial confirmatória eram abaixo do recomendável. No presente estudo também houve necessidade de descartar esses mesmos itens (Q1, Q5, Q7, Q12). A versão reduzida permite diferenciar os participantes de forma similar ao uso da escala com todos os itens, com a vantagem de avaliar com maior rigor as dimensões da eficácia e da satisfação parental.

Menéndez, Giménez e Hidalgo (2011) aplicaram a escala em 259 mães usuárias de serviços sociais comunitários na Espanha e pertencentes a famílias de baixa renda. Essas autoras também chegaram a uma versão reduzida da escala, de 10 itens, sendo que apenas dois dos itens excluídos (Q2 e Q14) não coincidem com os que foram excluídos nesta pesquisa. As autoras recomendam o uso da versão reduzida, para esse tipo de população. Como salientam Cutrona e Troutman (1986), as versões de instrumento mais curtas apresentam as vantagens da facilidade e rapidez na aplicação. Recomenda-se assim o uso desta versão reduzida, em alternativa à escala completa, para populações de baixa renda no Brasil.

Os resultados revelam que os fatores Eficácia e Satisfação não estão significativamente correlacionados. De modo semelhante Ferreira et al. (2011), encontraram correlação de 0,1 entre esses fatores. Mesmo em estudos que encontraram correlações maiores (Seabra-Santos et al., 2015), verifica-se que seus valores são de magnitude baixa a moderada (e.g., $d=0.39)$. Tais resultados apontam para a distinção entre as dimensões Eficácia e Satisfação (Ohan et al., 2000), assegurando a validade discriminante dos fatores da escala.

Neste estudo, os níveis apurados de eficácia foram significativamente mais altos do que os de satisfação, de modo semelhante ao encontrado por Nunes, Jiménez, Menéndez, Ayala-Nunes, \& Hidalgo (2016), que trabalharam em Portugal com mães de baixa renda. Entretanto há divergência com os achados em outros estudos (Ohan et al., 2000; Gomes-da-Costa et al., 2006). Como a amostra da presente pesquisa difere em termos de características socioeconômicas da maioria dos estudos realizados no exterior, recomenda-se a realização de novas pesquisas, a fim de verificar se tal resultado será replicado.

\section{Influência de fatores sociodemográficos}

Em relação à influência da idade dos pais sobre os níveis de satisfação e eficácia medidos pelo QACEP, as correlações não demostraram associações significativas. Contudo, a comparação entre diferentes grupos etários revela que os pais com mais idade (36-64 anos) apresentaram nível de satisfação significativamente maior do que os de menor idade (21-35 anos), enquanto nenhum efeito foi encontrado para a eficácia. Entretanto, o tamanho de efeito observado na diferença nos níveis de satisfação é muito pequeno, demonstrando não haver implicações práticas ou alterações no comportamento dos pais com maior idade decorrentes da sua maior satisfação. Outros estudos encontraram um efeito da idade apenas para a subescala de Eficácia. Gilmore e Cuskelly (2008) observaram que apenas os pais mais velhos, e não as mães, se avaliaram como menos eficazes que os mais novos. Uma associação negativa entre a idade e a eficácia foi também observada por Seabra-Santos et al. (2015) para o conjunto da amostra, composta maioritariamente por mulheres. Tais discrepâncias apontam a necessidade de novas pesquisas que ajudem a clarificar qual a relação da idade com a satisfação e a eficácia, sabendo que as dimensões da competência parental têm uma relação de baixa magnitude entre si.

Nesta pesquisa também se observou que o nível de escolaridade influiu sobre o nível de satisfação, pois os pais com escolaridade superior demonstraram mais satisfação parental do que os de escolaridade de nível médio e fundamental. De modo semelhante, no estudo de Seabra-Santos et al. (2015), observou-se uma correlação positiva e estatisticamente significativa entre os níveis de satisfação reportados e o número de anos de escolaridade completados. Também os estudos de Gilmores e Cuskely (2009) e de Trudelle e Montambaut (1994) revelaram que a satisfação aumenta com o nível educacional, ou seja, quanto maior o nível educacional, maior a satisfação.

Em relação às faixas salariais, esta pesquisa encontrou diferenças significativas na satisfação para pais que pertenciam a faixas salariais superiores em relação aos que ganhavam menos. A literatura internacional não tem relatado o efeito dessa variável. No Brasil, a pesquisa de Silva e Aiello (2009) revelou que os pais pertencentes aos níveis socioeconômicos mais elevados apresentaram resultados mais altos na pontuação total da escala do que os pais pertencentes a níveis inferiores. A esse 
respeito, deve-se levar em consideração que o aumento da faixa salarial possibilita aos pais acesso a uma série de recursos, como escolas em tempo integral, contratação de funcionários que os auxiliem com os cuidados aos filhos, condições que ajudam a diminuir sua carga de tarefas, o que pode repercutir nesses níveis mais elevados de satisfação. Outro ponto a ser considerado é que o nível econômico está relacionado com a escolaridade e, conforme já mencionado, a literatura tem mostrado que os níveis de satisfação são maiores para os pais com nível educacional mais alto. Todas as diferenças para a satisfação encontradas entre grupos adjacentes de idade, escolaridade e faixa salarial apresentam uma magnitude de tamanho pequeno, ou seja, o mínimo recomendado para avaliação de um efeito relevante em ciências sociais (Ferguson, 2009). Apenas a diferença observada entre o grupo com menor salário e o com maior salário é de magnitude moderada, o que possivelmente se traduz em diferenças que podem ser observadas nos comportamentos dos participantes.

Neste estudo, os níveis apurados de eficácia foram significativamente mais altos do que os de satisfação. Tal resultado diverge do encontrado na literatura (Ohan et al., 2000; Gomes-da-Costa et al., 2006). Como a amostra da presente pesquisa difere em termos de características socioeconômicas das que têm sido utilizadas em estudos no exterior, recomenda-se a realização de novos estudos, com amostras que apresentem características semelhantes, a fim de verificar se tal resultado será replicado.

\section{Limitações, implicações para a prática e considerações finais}

Uma das limitações deste estudo está relacionada com o baixo número de homens que respondeu ao QACEP. Tal fato pode estar relacionado com as condições de coleta de dados, que ocorreu em escolas, onde as mães costumam comparecer comparecer mais do que os pais. É conveniente que se busque um equilíbrio entre os sexos em próximas pesquisas que forem feitas com grupos que apresentem características semelhantes às do que foi aqui pesquisado, nomeadamente famílias de baixa renda.
Uma outra limitação prende-se à ausência da análise sobre o efeito da idade dos filhos dos participantes nas dimensões da competência parental, sendo que a literatura existente mostra que esta é uma variável que pode ter influência significativa na eficácia e satisfação parentais e que precisa ser melhor explorada (Gilmores \& Cuskely, 2009; Ohan et al., 2000; Rogers \& Mathews, 2004).

Deve-se ainda salientar que o QACEP possui itens construídos como afirmativas positivas (subescala de Eficácia) e outros construídos na negativa (subescala de Satisfação) e, como já salientaram Johnston e Mash (1989) e Ohan et al. (2000), a solução bi-fatorial pode estar refletindo essa valência, ou seja, aspectos positivos e negativos da parentalidade, mais do que aspectos da Eficácia e Satisfação. Considerando que a redação de itens na negativa pode dificultar o entendimento do enunciado, sugere-se que, em próximas pesquisas, essa característica da escala seja abordada, trabalhando-se exclusivamente com itens na afirmativa.

O QACEP é um instrumento que tem sido utilizado em amostras com níveis educacionais ou salariais superiores ao da amostra desta pesquisa (Cutrona \& Troutman, 1986; Gilmores \& Cuskely, 2009; Johnston \& Mash, 1989; Rogers \& Mathews, 2004). Assim, nesta pesquisa, procurou-se demonstrar que a escala pode ser utilizada num grupo de baixa renda. Adicionalmente, os procedimentos de adaptação ao contexto brasileiro demostram que a versão reduzida é mais rigorosa na aferição da percepção da eficácia e satisfação parental, pelo que se recomenda a utilização desta versão, em populações de baixa renda, no Brasil.

Tendo em vista a importância teórica e prática dos conceitos de Eficácia e Satisfação na qualidade do desempenho das funções parentais (Coleman \& Karraker, 1997, 2000; Jones \& Prinz, 2005), assim como na felicidade e bem-estar dos pais (Nelson, Kushlev, \& Lyubormirsky, 2014), deve-se salientar a utilidade do instrumento aqui pesquisado, que poderá servir para avaliar esses aspectos das competências parentais, no decorrer de intervenções clínicas com pais, voltadas para a melhoria das interações pais-filhos.

\section{Referências}

Afonso, A. F. L. (2011). A relação entre o sentimento de competência educativa parental, o apoio social, a autoestima e o rendimento escolar em alunos do ensino básico. (Dissertação de mestrado), Universidade Trás-os-Montes, Portugal, PT.

Associação Brasileira de Empresas de Pesquisa, ABEP (2016). Critério Brasil 2015 e atualização da distribuição de classes para 2016. Recuperado de http://www.abep.org/criterio-brasil

Brasil, Ministério do Trabalho e Emprego (2010). Classificação Brasileira de Ocupações, $3^{a}$ ed, Brasília.

Borsa, J. C., Damasio, B. F., \& Bandeira, D. R. (2012). Cross-cultural adaptation and validation of psychological instruments: Some considerations. Paideia, 22(53), 423-432. doi: 10.1590/S0103-863X2012000300014

Coleman, P.K., \& Karraker, K, H. (1997). Self-efficacy and parenting quality: Findings and future applications. Developmental Review, 18(1), 47-85. doi: 10.1006/drev.1997.0448

Coleman, P. K., \& Karraker, K, H. (2000). Parenting self-efficacy among mothers of school-age children: Conceptualization, measurement and correlates. Family Relations, 49(1), 13-24. doi: 10.1111/j.1741-3729.2000.00013.x 
Cutrona, C., \& Troutman, B. R. (1986). Social support, infant temperament, and parenting self-efficacy: A mediational model of postpartum depression. Child Development, 57(6), 1507-1518. doi: 10.2307/1130428

Ferguson, C. J. (2009) An effect size primer: A guide for clinicians and researchers. Professional Psychology: Research and Practice, 40 (5), $532-538$. doi: $10.1037 / \mathrm{a} 0015808$

Ferreira, B., Veríssimo, M., Santos, A J., Fernandes, C., \& Cardoso, J. (2011). Escala de sentimento de competência parental: Análise confirmatória do modelo de medida numa amostra de pais portugueses. Laboratório de Psicologia (ISPA), 9(2), 147-155. doi:10.14417/ lp. 630

Gilmore, L. A., \& Cuskelly, M. (2009). Factor structure of the parenting sense of competence Scale using a normative sample. Child Care, Health \& Development, 35(1), 48-55. doi: 10.1111/j.1365-2214.2008.00867.x

Gomes-da-Costa, J. G., Antunes, C., Além, A., Gouveia, L., Castro, E., \& Azevedo, C. (2011). Questionnaire d'autoévaluation de la competénce éducative parentale (Terrisse \& Trudele, 1998): application sur un échantillon de parents d'adolescents portugais. Em E. Catarsi \& J-P. Pourtois, J-P. (Eds.), XIII Congresso Internazionale - Educazione familiare e servizi per l'infanzia (Tomo primo) (pp.180-186). Italy: Firenze.

Grady, J. S., \& Karraker, K. (2016). Mother and child temperament as interacting correlates of parenting sense of competence in toddlerhood. Infant and Child Development, 26(4), 1-15. doi: 10.1002/icd.1997

Johnston, C., \& Mash, E.J. (1989). A measure of parenting satisfaction and efficacy. Journal of Clinical Child Psychology, 18(2), 167-175. doi: $10.1207 /$ s15374424jccp1802_8

Jones, T. L., \& Prinz, R. J. (2005) Potential roles of parental self-efficacy in parent and child adjustment: A review. Clinical Psychology Review, 25(3), 341-363. doi: 10.1016/j.cpr.2004.12.004

Marôco, J. (2010). Análise de equações estruturais. Fundamentos teóricos, software \& aplicações. Pêro Pinheiro, Portugal: Report Number.

Menéndez, S., Jiménez, L. \& Hidalgo, V. (2011) Estructura factorial de la escala PSOC (parental sense of competence) em una muestra de madres usuarias de servicios de preservación familiar. Revista Iberoamericana de Diagnóstico y Evaluación Psicológica, 32(2), $187-204$. Recuperado de: http://www.redalyc.org/articulo.oa?id=459645440009

Meunier, J. C., \& Roskam, I. (2009). Self-efficacy beliefs amongst parents of young children: Validation of a self-report measure. Journal of Child and Family Studies, 18(5), 495-511. doi: 10.1007/s10826-008-9252-8

Nelson, S. K., Kushlev, K., \& Lyubomirsky, S. (2014) Pains and pleasures of parenting: When, why and how is parenthood associated, with well-being? Psychological Bulletin, 140(3), 846-895. doi: 10.1037/a00354442009

Ngai, F., Chan, S.W., \& Holroyd, E. (2007). Translation and validation of a Chinese version of the Parenting Sense of Competence Scale in Chinese Mothers. Nursing Research, 56(5), 348-354. doi: 10.1097/01.NNR.0000289499.99542.94

Nunes, C., Jiménez, L., Menéndez, S., Ayala-Nunes, L., \& Hidalgo, V. (2016) Psychometric properties of an adapted version of the parental sense of competence (PSOC) scale for Portuguese at-risk parents. Child and Family Social Work, 22, 433-441. doi:10.1111/cfs.12159

Ohan, J., Leung, D. W., \& Johnston, C. (2000) The parenting sense of competence scale: Evidence of a stable factor structure and validity. Canadian Journal of Behavioural Science, 32(4), 251-261. doi:10.1037/h0087122

Rogers, H., \& Matthews, J. (2004). The parenting sense of competence scale: Investigation of the factor structure, reliability, and validity for an Australian sample. Australian Psychologist, 39(1), 88-96. doi: 10.1080/00050060410001660380

Seabra-Santos, M. J., Major, S., Pimentel, M., Gaspar, M. F. Antunes, N., \& Roque, V. (2015). Escala de Sentido de Competência Parental (PSOC): Estudos psicométricos. Avaliação Psicológica,14(1), 97-106. doi: 10.15689/ap.2015.1401.1

Silva, N. C. B., \& Aiello, A. L. R. (2009) Análise descritiva do pai da criança com deficiência mental. Estudos de Psicologia, Campinas, 26(4), 493-503. doi:10.1590/S0103-166X2009000400010

Terrisse, B., \& Trudelle, D. (1988) Le questionnaire d'auto-évaluation de la compétence educative parentale (QACEP). Université du Québec à Montreal, CN. Recuperado de https://unites.uqam.ca/terrisse/pdf/G29.pdf

Trudelle, D., \& Montambault, E. (1994). Le sentiment de compétence parentale chez des parents d'enfants en age préscolaire. Service Social, 43(2), 47-62. doi: 10.7202/706656ar

Wagner, F. R., \& Morse, J. J. (1975). A measure of individual sense of competence. Psychological Reports, 36(2), 451-459. Recuperado de http:// journals.sagepub.com/doi/abs/10.2466/pr0.1975.36.2.451

\section{Sobre as autoras}

Maria Benedita Lima Pardo é doutora em Psicologia Experimental pela Universidade de São Paulo e professora titular do Departamento de Psicologia da Universidade Federal de Sergipe.

Daniela Fonseca Freitas é doutora em Psicologia pela Faculdade de Psicologia e Ciências da Educação da Universidade do Porto e pela Faculdade de Filosofia, Ciências e Letras de Ribeirão Preto da Universidade de São Paulo e investigadora de pós-doutoramento no Centro de Psicologia da Universidade do Porto.

Margarida Silveira Britto de Carvalho é psicóloga pela UniCEUB, Brasília e professora adjunta do Departamento de Psicologia da Universidade Federal de Sergipe.

Anne Marie Fontaine é doutora em Psicologia e Professora Catedrática da Faculdade de Psicologia e Ciências da Educação da Universidade do Porto e coordenadora do projeto de pesquisa europeu INTERFASOL - (Intergenerational Family Solidarity across Europe). 